\title{
MOMENT INEQUALITIES OF THE LIAPUNOV TYPE
}

\author{
H. L. MACGILLIVRAY
}

(Received 17 January 1980)

Communicated by R. L. Tweedie

\begin{abstract}
Characterisations of the distribution of a non-negative random variable are sought for which the Liapunov moment inequality is extended to give inequalities between inverse powers of moment ratios, which are known as mean sizes in considerations of particle size distributions. A solution is found for continuous distributions, and the conditions applied to a number of well-known distributions. A further class of distributions is considered for which the new inequalities hold but the inequality direction is reversed for some orders of the moments. The study involves examination of the signs of the third central moments of a family of distributions, obtained by a log transformation, from the weighted, or moment, distributions induced by the non-negative random variable.
\end{abstract}

1980 Mathematics subject classification (Amer. Math. Soc.): primary 60 E 15; secondary 62 E 10.

\section{Introduction}

The Liapunov inequality for absolute moments essentially states that for any random variable, the natural logarithm of the $r$ th absolute moment is a convex function of $r$ (see for example, Moran (1968), p. 234). Hence, for a non-negative random variable whose $r$ th moment about the origin is denoted by $\alpha_{r}$ if it exists, $\log \alpha_{r}$ is a convex function of $r$, and thus $\left(\alpha_{r}\right)^{1 / r}$ is increasing in $r$. Further properties of $\log \alpha_{r}$ that are similar in concept have been established for particular classes of non-negative random variables. For Pólya densities of order 2 (Karlin, Proschan and Barlow (1961)), or, more generally, distributions with increasing hazard rate (Barlow, Marshall and Proschan (1963)), the log of the normalised moments is concave, giving that $\left(\alpha_{r} / \Gamma(r+1)\right)^{1 / r}$ is a decreasing function of $r$.

(C) Copyright Australian Mathematical Society 1981 
In this paper, the variation of $\left(\alpha_{r+u} / \alpha_{r-u}\right)^{1 / 2 u}$ in $u$ is examined, and characteristics of distributions sought for which $L_{r+u, r-u} \equiv\left(\alpha_{r+u} / \alpha_{r-u}\right)^{1 / 2 u}$ is monotonic in $u$. For continuous distributions, a characterisation is found that ensures $L_{r+u, r-u}$ is monotonic in $u$ for all possible values of $r$, and hence for these classes of continuous distributions, a new moment inequality is established which may be considered as an extension to the Liapunov inequality. A further class of distributions is then examined, for which $L_{r+u, r-u}$ is monotonic in $u$ for given $r$, but the values of $r$ fall into two subsets, in one of which $L_{r+u, r-u}$ is decreasing in $u$, and in the other, increasing. For these distributions, there is a value of $r$ that acts as a turning point and is a 'symmetry' value for the family of distributions with density given by $e^{(r+1) y} f\left(e^{y}\right) / \alpha_{r}$, where $f(x)$ is the density of the non-negative random variable.

The question of the variation of $L_{r+u, r-u}$ arose in consideration of particle size distributions in dispersed systems in chemical engineering. If the random variable, $X$, represents some measure of particle size, its $m, n$th mean size is defined by $L_{m, n}=\left(\alpha_{m} / \alpha_{n}\right)^{1(m-n)}$, and these mean sizes tend to be considered in engineering and related fields, rather than the moments themselves (White (1971), Allen (1975), Randolph and Larson (1971)). The ratio $\alpha_{m} / \alpha_{n}$ is the $(m-n)$ th moment of the weighted, or moment, distribution $F_{n}(x)=\int_{0}^{x} t^{n} / \alpha_{n} d F(t)$, where $F(x)$ is the distribution function of $X$. These weighted distributions have particular significance in various engineering and related fields, especially for $n<3$, and, for dimensional convenience, it is customary to use mean sizes (see, for example, Randolph and Larson (1971), White (1971)), especially in comparisons of system properties.

It follows immediately from the Liapunov inequality that $L_{m, n}$ is increasing in $m$ for fixed $n$, and increasing in $n$ for fixed $m$, and the request for information regarding 'diagonal' relationships between mean sizes led to the work presented in this paper.

\section{Some preliminary remarks}

The Liapunov inequality may be proved by considering moments as differentiable functions of their orders and showing that the second derivative is non-negative; this was noted by Belz (1947). However, the work reported in Belz's note did not require a precise expression of the range on which $\alpha_{r}$ is differentiable, and since differentation of $\alpha_{r}$ is used extensively in this paper, the following lemma is required.

LEMMA 1. If the absolute moments of order $r, \nu_{r}$, of a random variable with distribution function $F(x)$ exist for $r$ in the interval $[0, b]$, then $\nu_{r}$ is differentiable 
with respect to $r$ on $(0, b)$, and the nth derivative is given by

$$
\frac{d^{n} \nu_{r}}{d r^{n}}=\int_{-\infty}^{\infty}(\log |x|)^{n}|x|^{r} d F(x)
$$

The proof follows readily from dominated convergence, with the absolute value of the difference quotients being bounded above by an integrable function of $x$ for all $t$ in $(0, b)$.

Lemma 2 states some properties of convex and concave functions in forms which are required in this paper.

LEMMA 2. If the first derivative of the real function $\rho(t)$ is convex (concave), then

(a) $(\rho(t+u)-\rho(t-u)) / 2 u$ is increasing (decreasing) in $u$ for any $t$, and this condition is necessary and sufficient; and

(b) $\rho(t+u)-\rho(t-u)$ has at most one change of sign in $u>0$, and if there is a change of sign, it is from negative to positive values (positive to negative).

Proof. (a)

$$
\begin{aligned}
& \frac{d}{d u}\left(\frac{\rho(t+u)-\rho(t-u)}{2 u}\right) \\
& \quad=\frac{1}{2 u^{2}} \int_{0}^{u}\left[\rho^{\prime}(t+u)+\rho^{\prime}(t-u)-\rho^{\prime}(t+x)-\rho^{\prime}(t-x)\right] d x
\end{aligned}
$$

For $\rho^{\prime}(x)$ convex (concave) for all $x$ and $x<u$, the integrand is non-negative (non-positive), and hence necessity is proved.

If $(2.2)$ is $>0(\leqslant 0)$ for all $u, t$, suppose there is some interval on which $\rho^{\prime}(x)$ is not convex (concave). Then for some $t$ and some $u>0$, the integrand in (2.2) is $<0(>0)$, giving a contradiction.

(b) Follows directly from (a) since $u>0$.

Corollary 2.1. If $\rho^{\prime}(t)$ is linear, then $(\rho(t+u)-\rho(t-u)) / 2 u$ is constant, and $\rho(t+u)-\rho(t-u)$ is a multiple of $u$.

\section{A new moment inequality}

From Lemmas 1 and 2

$$
L_{r+u, r-u}=\left(\frac{\alpha_{r+u}}{\alpha_{r-u}}\right)^{1 / 2 u}
$$


is an increasing function of $u$ if, and only if, $\left(\log \alpha_{r}\right)^{(3)}>0$ for all $r>0$. Hence, for $0<u<v<r$,

$$
\left(\frac{\alpha_{r+u}}{\alpha_{r-u}}\right)^{1 / 2 u} \leqslant\left(\frac{\alpha_{r+v}}{\alpha_{r-v}}\right)^{1 / 2 v},
$$

which may be rewritten as

$$
\alpha_{r+u}^{v} \alpha_{r-v}^{u} \leqslant \alpha_{r-u}^{v} \alpha_{r+v}^{u} .
$$

$L_{r+u, r-u}$ is decreasing in $u$ if and only if $\left(\log \alpha_{r}\right)^{(3)} \leqslant 0$ for all $r>0$, and the inequalities (3.1) and (3.2) are reversed.

These can be compared with the Liapunov inequality which corresponds to $\left(\log \alpha_{r}\right)^{(2)} \geqslant 0$, and $\left(\alpha_{r}\right)^{1 / r}$ increasing in $r$, and which gives for $0<u<v<r$,

$$
\alpha_{r-u} \alpha_{r+u} \leqslant \alpha_{r+v} \alpha_{r-v} .
$$

The inequalities (3.1) and (3.2) will be referred to as inequality $I_{1}$, and the reverse inequalities corresponding to $\left(\log \alpha_{r}\right)^{(3)}<0$, as inequality $I_{2}$. As will be seen in Table $1, I_{2}$ holds more frequently than $I_{1}$.

Denoting $X_{r}$ as the random variable with distribution $F_{r}(x), Y_{r}=\log X_{r}$ is well-defined for all possible $r$, with distribution function

$$
G_{r}(y)=\int_{-\infty}^{y} \frac{e^{r t}}{\alpha_{r}} d F\left(e^{t}\right) .
$$

The ratio $\left(\alpha_{r+s} / \alpha_{r}\right)$, the $s$ th moment of $X_{r}$, is the moment generating function about the origin of $Y_{r}$.

The $n$th cumulant of $Y_{r}$ is given by

$$
\left.\frac{d^{n}}{d s^{n}}\left[\log \frac{\alpha_{r+s}}{\alpha_{r}}\right]\right|_{s=0}=\frac{d^{n}}{d r^{n}}\left(\log \alpha_{r}\right)
$$

and thus $\left(\log \alpha_{r}\right)^{(3)}$ is the third central moment of $Y_{r}$. Hence if the 'base' distribution $F(x)$ is such that the third central moments of all the members of the family $\left\{G_{r}(y)\right\}$ are of the same sign, then either inequality $\mathrm{I}_{1}$ or $\mathrm{I}_{2}$ will hold for all values of $r, u$ and $v$ for which the moments exist. Theorem I gives a condition on the distribution of a continuous random variable, sufficient for the third central moment to be of a given sign.

Although Theorem $I$ is more appropriate for density functions whose support is the whole real line, some generalisation is possible to those whose support is a subset of the real line. This is discussed after Theorem $I$ and the application of the general case to the family $\left\{g_{r}(y)\right\}$ summarised in Theorem II, where $g_{r}(y)$ is the density corresponding to $G_{r}(y)$.

The proof of Theorem I uses the variation diminishing property of totally positive kernels in its stronger form (Karlin (1968), pp. 20, 21) in which the following definitions of the number of changes of sign of a function are used. 
Let $k(t)$ be defined in $I$, where $I$ is an ordered set of the real line. Let

$$
S^{-}(k)=S^{-}[k(t)]=\sup S^{-}\left[k\left(t_{1}\right), k\left(t_{2}\right), \ldots, k\left(t_{m}\right)\right],
$$

where the supremum is extended over all sets $t_{1}<t_{2}<\cdots<t_{m}\left(t_{i} \in I\right), m$ is arbitrary but finite, and $S^{-}\left(x_{1}, x_{2}, \ldots, x_{m}\right)$ is the number of sign changes of the indicated sequence, zero terms being discarded. A more refined method of counting sign changes is given by

$$
S^{+}(k)=S^{+}[k(t)]=\sup S^{+}\left[k\left(t_{1}\right), k\left(t_{2}\right), \ldots, k\left(t_{m}\right)\right],
$$

where $S^{+}\left(x_{1}, \ldots, x_{m}\right)$ denotes the maximum number of sign changes of the indicated sequence, the zero terms being permitted to take on arbitrary signs.

THEOREM I. If $V$ is a continuous random variable defined on $(-\infty, \infty)$, with a differentiable probability density function $h(v)$, mean $\mu$ and sth central moment $\mu_{s}$, then if either $h^{\prime}(v)$ or $(\log h(v))^{\prime}$ is convex (concave) for all $v$ (but not linear), $\mu_{3}$ is positive (negative).

Proof. (a) For $s$ odd,

$$
\mu_{s}=\int_{0}^{\infty} t^{s}[h(\mu+t)-h(\mu-t)] d t .
$$

For $h^{\prime}(v)$ convex, it follows from Lemma 2(b) that $h(\mu+t)-h(\mu-t)$ has at most one change of sign in $t$, from negative to positive values. Since $t^{s}$ is totally positive (TP) for $s, t>0$ (Karlin and others (1961)), from the variation diminishing property, it follows that (3.5) has at most one change of sign in $s$, from negative to positive values. Indeed, $t^{s}$ is strictly totally positive and hence, provided $h(\mu+t)-h(\mu-t)$ is not identically zero, the stronger version of the variation diminishing property holds, and

$$
S^{+}(3.5) \leqslant S^{-}[h(\mu+t)-h(\mu-t)]<1,
$$

where $S^{+}$and $S^{-}$are as defined in (3.3) and (3.4). Since $s=1$ is a zero of (3.5), $S^{+}(3.5) \geqslant 1, h(\mu+t)-h(\mu-t)$ must change sign, and $S^{+}(3.5)=1$. The zero of (3.5) at $s=1$ must also be a change of sign otherwise $S^{+}(3.5)=2$, as in counting the sign changes $S^{+}$, the zero term may taken on an arbitrary sign. Therefore (3.5) changes sign once at $s=1$ from negative to positive values, and the third central moment $\mu_{3}$ is positive.

Similarly, for $h^{\prime}(v)$ concave, $\mu_{3}$ is negative provided $h(\mu+t)-h(\mu-t)$ is not identically zero.

Since the $\log$ function is monotonic increasing, the condition on $\log h(v)$ follows.

The case $h(\mu+t)-h(\mu-t)$ identically zero, and hence $\mu_{3}=0$, corresponds in this theorem to the degenerate case of $h^{\prime}(v)$ or $(\log h(v))^{\prime}$ linear for all $v$. 
Linearity implies that $h(\mu+t)-h(\mu-t)$ does not change sign for $t>0$ (Corollary 2.1), and since at least one change of sign is necessary for $\mu$ of equation (3.5) to be zero, $h(\mu+)-h(\mu-t)$ must be identically zero, that is, $h(v)$ symmetric about its mean. For a concave or convex derivative as assumed in this theorem,

$$
h(\mu+t)-h(\mu-t) \equiv 0
$$

in $t$ implies linearity of the appropriate derivative. Linearity of $h^{\prime}(v)$ for all $v$ is impossible and the truncated range case is discussed below; linearity of $(\log h(v))^{\prime}$ corresponds to the normal distribution.

REMARK. For an arbitrary random variable $V$ with distribution function $H(v)$, the central moments $\mu_{s}$ may be written as

$$
\mu_{s}=s \int_{0}^{\infty} t^{s-1}[1-H(\mu+t)-H(\mu-t)] d t .
$$

If it is known, or can be proved, that the number of changes of sign of [1-H( $\mu+t)-H(\mu-t)]$ is bounded by 1 , then the reasoning of Theorem I applies, and the sign of $\mu_{3}$ may be determined. This is not pursued further here.

REMARK. The totally positive properties of $t^{s}$ are a feature of the proof of the normalised moments inequality due to Karlin, Proschan and Barlow (1961) mentioned in Section 1. The kernel $t^{s}$ has also been used in moment comparisons for two symmetric distributions (Marsaglia, Marshall and Proschan (1965)).

Applying the condition on the $\log$ of the density to $g_{r}(y)$, gives that if $d\left[\log f\left(e^{y}\right)\right] / d y$ is concave (convex) in $y$, then inequality $I_{2}\left(I_{1}\right)$ holds.

Theorem I may be generalised by considering equation (3.5) and seeking conditions for which $h(\mu+t)-h(\mu-t)$ has at most one change of sign in $t>0$. Use is also made of the requirement that $h(\mu+t)-h(\mu-t)$ must have at least one change of sign to ensure $\mu_{1}=0$, since the stronger version of the variation-diminishing property holds. Consideration of (3.5) when the support of the probability density function $h(v)$ is some interval $(a, b)$ shows that if $h(v)$ is continuous at $a$ and $b$, then Theorem I holds with the condition of convexity (concavity) of $h^{\prime}(v)$ or $(\log h(v))^{\prime}$ applying only in the interval $(a, b)$. Some results can also be obtained when $h(v)$ is not necessarily continuous at $a$ and $b$. The concave and convex cases are considered separately.

When $d h(v) / d v$ or $d(\log h(v)) / d v$ is concave in $(a, b)$ :

If $h(\mu+t)-h(\mu-t)$ changes sign for $(\mu-t, \mu+t)$ contained in $(a, b)$, it is from positive to negative values. Therefore, if $a=-\infty$, and $b<\infty$, no further change of sign occurs, but if $a>-\infty, b=\infty$, there is a second change of sign from negative to positive values at $\mu-t=a$. 
When $d h(v) / d v$ or $d(\log h(v)) / d v$ is convex in $(a, b)$ :

As above, if $h(\mu+t)-h(\mu-t)$ changes sign for $a<\mu-t<\mu+t<b$, it is from negative to positive values. Therefore if $a>-\infty, b=\infty$, no further change of sign occurs, but if $a=-\infty, b<\infty$, a second change of sign from positive to negative values takes place at $\mu+t=b$.

When both $a$ and $b$ are finite, the distribution requires special examination. In applications to the family $\left\{g_{r}(y)\right\}$, this involves examining $g_{r}\left(\eta_{r}+y\right)-$ $g_{r}\left(\eta_{r}-y\right)$ directly, where $\eta_{r}=\left(\log \alpha_{r}\right)^{\prime}$ is the mean of $g_{r}(y)$.

If it is known that $h(\mu+t)-h(\mu-t)$ does not change sign for $a<\mu-t$ $<\mu+t \leqslant b$, whether $h^{\prime}(v)$ or $(\log h(v))^{\prime}$ is concave, convex or neither, then at most one change of sign will occur at $t=\min (b-\mu, \mu-a)$. Since in (3.5) $\mu_{1}=0$, and the stronger version of the variation-diminishing property holds, $h(\mu+t)-h(\mu-t)$ must change sign at least once. Therefore, if $h(\mu+t)-$ $h(\mu-t)$ is non-negative inside $(a, b)$, the change is from positive to negative values and $\mu_{3}<0$ : if it is non-positive in $(a, b), \mu_{3}>0$; and if $h(\mu+t)-$ $h(\mu-t)$ is identically zero, $\mu_{3}=0$. If the support is $(-\infty, b)$, a change of sign will occur only if $h(\mu+t)-h(\mu-t)$ is non-negative in $(-\infty, b)$, and thus $\mu_{3}<0$. Similarly, if the support is $(a, \infty), h(\mu+t)-h(\mu-t)$ must be nonpositive in $(a, \infty)$ and thus $\mu_{3}>0$.

A special case of the above occurs when $h(v)$ is monotonic in $(a, b)$. Another special case corresponds to $h^{(3)}(v)$ or $(\log h(v))^{(3)}$ identically zero in $(a, b)$, for then $h(\mu+t)-h(\mu-t)$ is of one sign in $(a, b)$; the lower order derivatives of $h(v)$ determine the sign.

As before, in applying the above to the family $\left\{g_{r}(y)\right\}$, the conditions on $\log h(v)$ are used, to give conditions on the base distribution $f(x)$ sufficient for $\left(\log \alpha_{r}\right)^{(3)}$ to be of one sign for all values of $r$, and hence $L_{r+u, r-u}$ monotonic in $u$ for all $r$ for which the moments exist. Theorem II states the results.

THEOREM II. Suppose $X$ is a continuous non-negative random variable with a probability density function $f(x)$ with support $[c, d]$ and differentiable in $(c, d) ; \alpha_{r}$ is the rth moment about the origin. Then

(a) if $\left(\log f\left(e^{y}\right)\right)^{\prime}$ is convex in $(\log c, \log d)$, and provided $f(x)$ is continuous at $d$ for $d<\infty,\left(\log \alpha_{r}\right)^{(3)}>0,\left(\alpha_{r+u} / \alpha_{r-u}\right)^{1 / 2 u}$ is strictly increasing in $u$;

(b) if $\left(\log f\left(e^{y}\right)\right)^{\prime}$ is concave in $(\log c, \log d)$, and provided $f(x)$ is continuous at $c$ for $c>0,\left(\log \alpha_{r}\right)^{(3)}<0$ and $\left(\alpha_{r+u} / \alpha_{r-u}\right)^{1 / 2 u}$ is strictly decreasing in $u$;

(c) if $\left(\log f\left(e^{y}\right)\right)^{\prime}$ is linear in $(\log c, \log d)$, either

(i) for $f(x)$ continuous at $c$ and $d,\left(\log \alpha_{r}\right)^{(3)} \equiv 0$ and $\left(\alpha_{r+u} / \alpha_{r-u}\right)^{1 / 2 u}$ is constant;

(ii) for $f(x)$ continuous at $c$ but not $d,\left(\log \alpha_{r}\right)^{(3)}<0$ and $\left(\alpha_{r+u} / \alpha_{r-u}\right)^{1 / 2 u}$ is decreasing in $u$; 
(iii) for $f(x)$ continuous at $d$ but not $c,\left(\log \alpha_{r}\right)^{(3)}>0$ and $\left(\alpha_{r+u} / \alpha_{r-u}\right)^{1 / 2 u}$ is increasing in $u$.

\begin{tabular}{|c|c|c|}
\hline $\begin{array}{l}\text { Behaviour of } \\
L_{r+u, r-u}\end{array}$ & $\begin{array}{l}\text { Distribution and Form } \\
\text { of Density Function }\end{array}$ & \\
\hline $\begin{array}{l}\text { Strictly } \\
\text { decreasing }\end{array}$ & $\begin{array}{l}\text { Pearson Type I; } x^{m_{1}}(a-x)^{m_{2}}, \\
\text { Pearson Type III; } x^{m e^{-x / c}} \\
\text { Weibull; } \frac{(x)}{\alpha}{ }^{c-1} \exp -\frac{(x)}{\alpha}^{c}, \\
\text { Planck's; } \frac{x^{f}}{e^{x}-1}, \\
\text { Linear failure rate; } \\
\text { (1 + } \theta x) \text { exp }-\left(x+\frac{\theta x^{2}}{2}\right) \\
\text { Normal with negative values } \\
\text { truncated; exp }-\frac{(x-\mu)^{2}}{2 \sigma^{2}}, \\
\text { Except for Pearson Type I, each of } \\
\text { the above distributions when } \\
\text { truncated on the right. }\end{array}$ & $\begin{array}{r}m_{1}, m_{2}>-1, a>0 \\
0<x<a . \\
m>-1, c>0 \\
0<x<\infty \\
c, \alpha>0, \\
0<x<\infty . \\
f>0,0<x<\infty . \\
\theta<1,0<x<\infty \\
\mu<0, \sigma^{2}>0 \\
0<x<\infty\end{array}$ \\
\hline $\begin{array}{l}\text { Strictly } \\
\text { increasing }\end{array}$ & Pearson Type V; $x^{-1 / c_{e}-c x}$, & $\begin{array}{l}\qquad c>0,0<x<\infty \text {, } \\
\text { and when truncated on the left. }\end{array}$ \\
\hline Increasing & $\begin{array}{l}\text { Pareto; } a k^{a} / x^{a+1} \text {, } \\
\text { (Strictly increasing when truncated } \\
\text { on right). }\end{array}$ & $a, k>0, k<x<\infty$ \\
\hline Constant & $\begin{array}{l}\text { Lognormal; } \frac{1}{x} \exp -\frac{(\log x-\xi)^{2}}{2 \sigma^{2}} \text {, } \\
\text { (Increasing when distribution } \\
\text { truncated on the left, } \\
\text { decreasing when distribution } \\
\text { truncated on the right). }\end{array}$ & $\begin{array}{r}-\infty<\xi<\infty, \sigma^{2}>0 \\
0<x<\infty .\end{array}$ \\
\hline
\end{tabular}


Some of the special cases discussed in generalising Theorem I are not covered by Theorem II. For example, if $g_{r}(y)$ is monotonic in $(\log c, \log d)$, the sign of $\left(\log \alpha_{r}\right)^{(3)}$ is determined as follows: for $g_{r}(y)$ increasing in $y$ for any $r,\left(\log \alpha_{r}\right)^{(3)}$ $<0$; for $g_{r}(y)$ decreasing in $y$ for any $r,\left(\log \alpha_{r}\right)^{(3)}>0$; while for $g_{r}(y)$ constant in $y,\left(\log \alpha_{r}\right)^{(3)} \equiv 0$.

Table 1 gives examples of density functions $f(x)$ of nonnegative random variables for which $L_{r+u, r-u}$ is monotonic; where a result exists for a truncation of $f(x)$, this is also stated.

\section{Symmetry values of $r$ for the distribution family $\left\{g_{r}(y)\right\}$}

From (2.2), $L_{r+u, r-u}$ is an increasing function of $u$ if $(r-u, r+u)$ lies in an interval in which $\left(\log \alpha_{t}\right)^{\prime}$ is convex, and is decreasing when $\left(\log \alpha_{t}\right)^{\prime}$ is concave. However, no general conclusions can be drawn if, in $(r-u, r+u),\left(\log \alpha_{t}\right)^{\prime}$ is both convex and concave, so that even if the exact behaviour of $\left(\log \alpha_{t}\right)^{\prime}$ is known, this is not sufficient in itself to describe the variation of $L_{r+u, r-u}$.

For certain distributions, however, although $\left(\log \alpha_{t}\right)^{\prime}$ changes from concave to convex or conversely, the behaviour of $L_{r+u, r-u}$ can be described exactly. For these distributions, there is a unique value of $r$ for which $g_{r}(y)$ is symmetric, and which acts as a turning point in the monotonic behaviour of $L_{r+u, r-u}$. Theorem III and Corollary III.1 give general results, but the application of these results implicitly depends on the solution of a problem that is possibly unsolvable in a general context; namely the problem of characterising distributions for which a zero third central moment implies that the distribution is symmetric. However, Theorem III can be applied to certain classes of distributions without the general problem being solved, and this is discussed in Section 5.

THEOREM III. In the interval of existence of the moments $\alpha_{r}$, there exists a value of $r, c$, such that $L_{r+u, r-u}$ is increasing in $u$ for $r<c$ and decreasing in $u$ for $r>c$ (or conversely), if and only if $G_{c}(y)$ is symmetric about its mean, and for no other possible value of $r$ does $\left(\log \alpha_{r}\right)^{(3)}$ change sign.

ProOF. (a) Necessity. Let $\eta_{c}$ be the mean of $G_{c}(y)$, that is,

$$
\eta_{c}=\left.\frac{d}{d r}\left(\log \alpha_{r}\right)\right|_{r=c}
$$

Suppose $G_{c}(y)$ is symmetric about $\eta_{c}$. Then

$$
E\left[\exp \left(Y_{c}-\eta_{c}\right) u\right]=E\left[\exp \left(\eta_{c}-Y_{c}\right) u\right],
$$


for $u$ such that these expectations exist. That is,

$$
e^{-\eta_{c} u} \frac{\alpha_{c+u}}{\alpha_{c}}=e^{\eta_{c} u} \frac{\alpha_{c-u}}{\alpha_{c}}
$$

defining $\psi(r) \equiv \log \alpha_{r},(4.1)$ is rewritten

$$
\psi(c+u)=2 u \psi^{\prime}(c)+\psi(c-u) .
$$

Successive differentation of (4.2) with respect to $u$, gives for $n>2$,

$$
\psi^{(n)}(c+u)=(-1)^{n} \psi^{(n)}(c-u),
$$

where

$$
\left.\psi^{(n)}(t) \equiv \frac{d^{n}}{d r^{n}} \psi(r)\right|_{r \rightarrow-i}
$$

Since $\psi^{(3)}(c+u)=-\psi^{(3)}(c-u), \psi^{(3)}(r)$ must change sign at $r=c$ or be identically zero for all possible $r$, and since it is assumed that $c$ is the only value of $r$ for which $\psi^{(3)}(r)$ may change sign, one of the following cases holds:

(i) if $\psi^{(4)}(c)>0, \psi^{(3)}(r) \leqslant 0$ for $r<c$,

$$
\text { and } \psi^{(3)}(r) \geqslant 0 \text { for } r>c \text {; }
$$

(ii) if $\psi^{(4)}(c)<0$, the inequalities (4.4) are reversed.

Considering case (i), if $r<c$, and $0 \leqslant v \leqslant r$, then for $r+v \leqslant c$,

$$
\psi^{(2)}(r-v) \geqslant \psi^{(2)}(r+v) \text {, }
$$

while for $r+v \geqslant c$

$$
\begin{aligned}
\psi^{(2)}(r-v) & \geqslant \psi^{(2)}(c-v)=\psi^{(2)}(c+v) \\
& \geqslant \psi^{(2)}(r+v) .
\end{aligned}
$$

Therefore, for $r<c$ and $0 \leqslant v \leqslant r$,

$$
\psi^{(2)}(r-v) \geqslant \psi^{(2)}(r+v) .
$$

Integrating (4.5) with respect to $v$ from $x$ to $u \leqslant r$ gives

$$
\psi^{\prime}(r-x)-\psi^{\prime}(r-u) \geqslant \psi^{\prime}(r+u)-\psi^{\prime}(r+x),
$$

and since

$$
\begin{aligned}
& \frac{d}{d u} \log L_{r+u, r-u} \\
& \quad=\frac{1}{2 u^{2}} \int_{0}^{u}\left[\psi^{\prime}(r+u)+\psi^{\prime}(r-u)-\psi^{\prime}(r+x)-\psi^{\prime}(r-x)\right] d x,
\end{aligned}
$$

$L_{r+u, r-u}$ is decreasing in $u$ for $r<c$. Similarly, it can be shown that $L_{r+u, r-u}$ is increasing in $u$ for $r>c$, and in case (ii) the inequalities are reversed. It is obvious that if $\psi^{(3)}(r)$ is identically zero for all possible values of $r, L_{r+u, r-u}$ will be constant in $u$ for all possible $r$. 
(b) Sufficiency. Suppose $L_{r+u, r-u}$ is increasing in $u$ for $r<c$ and decreasing in $u$ for $r>c$. That is,

$$
\begin{aligned}
\frac{d}{d u}\left(\log L_{r+u, r-u}\right) & >0, \text { for } r<c, \text { and } \\
& <0, \text { for } r>c .
\end{aligned}
$$

Since $\alpha_{r}$ is continuously differentiable, $d\left(\log L_{r+u, r-u}\right) / d u$ is continuous in $r$, and therefore

$$
\frac{d}{d u}\left(\log L_{c+u, c-u}\right)=0
$$

for all possible $0<u<c$.

Since

$$
\begin{aligned}
& \frac{d}{d u}\left(\log L_{c+u, c-u}\right)=\frac{\psi^{\prime}(c+u)+\psi^{\prime}(c-u)}{2 u}-\frac{\psi(c+u)-\psi(c-u)}{2 u^{2}} \\
& \begin{aligned}
\psi^{\prime}(c+u)+\psi^{\prime}(c-u) & =\frac{\psi(c+u)-\psi(c-u)}{u} \\
& =2 \log L_{c+u, c-u} \\
& =\text { constant for } 0<u<c .
\end{aligned}
\end{aligned}
$$

Successive differentiation of (4.6) with respect to $u$ gives $\psi^{(n)}(c+u)=$ $(-1)^{n} \psi^{(n)}(c-u), n>2,0<u<c$ as in (4.3).

Since $\psi(r)$ is continuously differentiable, from (4.3) it follows that for $n$ odd, $\psi^{(n)}(c)=0$, that is, the odd central moments of $G_{c}(y)$ are zero.

The characteristic function, $\varphi_{c}(t)$, of the distribution $G_{c}(y)$ is analytic, since there exists a positive constant $h=\min (c, b-c)$, where $[0, b]$ is the interval of existence of the moments $\alpha_{r}$, such that, for $s<h, 1-G_{c}(y)+G_{c}(-y)=o\left(e^{-v y}\right)$ as $y \rightarrow \infty$ (Lukacs (1960), p. 137). Hence $\varphi_{c}(z)$ can be expanded as an infinite series in $z$, convergent for $|z|<$ some $\rho>0$, and therefore $G_{c}(y)$ is uniquely determined by its moments. Since its odd central moments are zero, $G_{c}(y)$ is symmetric about its mean.

Suppose there exists another value of $r, c_{1}$, such that $\psi^{(3)}(r)$ changes sign at $r=c_{1}$, and suppose $c_{1}<c$. Then for $r_{1}<c_{1}<r<c$, and $u$ in some neighbourhood of zero, one of $L_{r+u, r-u}$ will be increasing in $u$ and the other decreasing. This contradicts the assumption of the theorem, and there is no other value of $r$ for which $\psi^{(3)}(r)$ changes sign.

The proof for the case $L_{r+u, r-u}$ decreasing in $u$ for $r<c$, and increasing for $r>c$, is exactly similar.

The values of $r$ for which $G_{r}(y)$ is symmetric will be referred to as symmetry values. From Theorem III, the following corollary is derived. 
COROLLARY III.1. If the family $\left\{G_{r}(y)\right\}$ has more than one symmetry value, then the symmetry values either form an arithmetic progression or consist of all values of $r$. Therefore if all the moments $\alpha_{r}$ exist, either none, one or an infinite number of values of $r$ are symmetry values.

Proof. From the proof of Theorem III, it can be seen that the system of equations (4.3), namely

$$
\psi^{(u)}(c+u)=(-1)^{n} \psi^{(n)}(c-u), \quad n>2,
$$

is necessary and sufficient for $c$ to be a symmetry value of $\left\{G_{r}(y)\right\}$.

Suppose $c+a$ is another symmetry value and consider $c+2 a, c-a$, provided these are possible orders of the moments $\alpha_{r}$. Then

$$
\begin{aligned}
\psi^{(n)}(c-a+u) & =(-1)^{n} \psi^{(n)}(c+a-u), \quad(c \text { a symmetry value }), \\
& =\psi^{(n)}(c+a+u), \quad(c+a \text { a symmetry value }), \\
& =(-1)^{n} \psi^{(n)}(c-a-u),
\end{aligned}
$$

and therefore $c-a$ is also a symmetry value. Similarly $c+2 a$ is a symmetry value.

If another symmetry value $c+b$ exists, where $b$ is not a multiple of $a$, the arithmetic progression of symmetry values will have a smaller difference, the actual magnitude depending on the relative magnitudes of $a, b,|a-b|$.

The set of symmetry values for the family induced by the log-normal distribution consists of all values of $r$. It was noted in Section 3 that the distribution $g_{r}(y)$ induced by the log-normal density function $f(x)$ where

$$
f(x) \alpha \frac{1}{x} \exp -\frac{(\log x-\xi)^{2}}{2 \sigma^{2}}, \quad x>0,
$$

is symmetric for all $r>0$. The constant value of $L_{r+u, r-u}$ in this case is given by $\exp \left(\xi+r \sigma^{2}\right)$.

\section{Application of Theorem III}

This theorem as it stands appears difficult to apply because either the form of $\log \alpha_{r}$ must be amenable to analysis, which is seldom the case, or it must be known that the family $G_{r}(y)$ is such, that if the third central moment of $Y_{r}$ is zero, its distribution is symmetric about its mean. This problem in a general context is as yet unsolved and a complete solution may not exist. However, a general solution is not necessary for the application of Theorem III to certain types of continuous distributions.

It is often straightforward to find possible symmetry values of $G_{r}(y)$ when the base distribution $F(x)$ is a continuous distribution. If the base density function 
$f(x)$ is differentiable in its support, a necessary condition for $g_{r}(y)$ to be symmetric about its mean, $\eta_{r}=\left(\log \alpha_{r}\right)^{\prime}$, is that

$$
\left.\frac{d^{2}}{d t^{2}}\left(\log f\left(e^{t}\right)\right)\right|_{t=\eta_{r}+y}=\left.\frac{d^{2}}{d t^{2}}\left(\log f\left(e^{t}\right)\right)\right|_{t=\eta_{r}-y}
$$

for all $y \geqslant 0$ such that

$$
f\left(e^{\eta, \pm y}\right)>0 .
$$

Denoting the function $\log f\left(e^{y}\right)$ by $l(y)$, a symmetry point $\gamma$ must satisfy

$$
l^{(2)}(\gamma+y)=l^{(2)}(\gamma-y) \text { where } f\left(e^{\gamma \pm y}\right)>0 .
$$

As in Corollary III.1, if there is more than one such $\gamma$, they either form an arithmetic progression or consist of all values of $y$ such that $f\left(e^{y}\right)>0$. If $f(x)>0$ on $(0, \infty)$, there is therefore none, one or an infinite number of $\gamma$ 's satisfying (5.2).

A value $\gamma$ that satisfies (5.2) can correspond to a symmetry value of $g_{r}(y)$ only if it is a permissible value of $\eta_{r}$. If this is so, $g_{r}(\gamma+y)-g_{r}(\gamma-y)$ is either identically zero or a multiple of $y$. As in Section 3 , if $f(x)$ is continuous on $(0, \infty)$, the latter case is inadmissible since $g_{r}(\gamma+y)-g_{r}(\gamma-y)$ must have at least one change of sign in $y$ for $\gamma$ to be the mean of $g_{r}(y)$.

If $S^{-}\left(g_{r}\left(\eta_{r}+y\right)-g_{r}\left(\eta_{r}-y\right)\right)<1$, then only for symmetry values does $\left(\log \alpha_{r}\right)^{(3)}=0$. In this case if there is just one value of $\gamma$ satisfying (5.2), either it corresponds to the unique symmetry value and Theorem III applies, or if it does not correspond to any $\eta_{r}$, $\left(\log \alpha_{r}\right)^{(3)}$ does not change sign and either inequality $I_{1}$ or $\mathrm{I}_{2}$ hold for all possible $r$.

For the case $f(x)$ continuous on $(0, \infty)$, Section 3 gives results when $\left(\log f\left(e^{x}\right)\right)^{(3)}$ does not change sign. When $\left(\log f\left(e^{x}\right)\right)^{(3)}$ changes sign once the following lemma gives a condition which enables Theorem III to be applied.

LEMMA 3. If, for the probability density function $f(x)$ defined and continuous on $(0, \infty),\left(\log f\left(e^{y}\right)\right)^{(3)}$ exists and changes sign once, and there exists a point $\gamma$ satisfying (5.2), then provided $\left(\log f\left(e^{y}\right)\right)^{(2)}$ tends to a finite limit as $y \rightarrow \infty$, $g_{r}(\eta+y)-g_{r}(\eta-y)$ has only one change of sign in $y>0$ for all $\eta \neq \gamma$, and thus Theorem III is applicable.

PRoof. With the notation $l(y)=\log f\left(e^{y}\right)$, for $\eta>\gamma$,

$$
\begin{aligned}
l^{(2)}(\eta+y)-l^{(2)}(\eta-y) & =l^{(2)}(\eta+y)-l^{(2)}(2 \gamma+y-\eta) \\
& =\int_{2 \gamma+y-\eta}^{\eta+y} l^{(3)}(t) d t, \\
& =\int_{-\infty}^{\infty} K(y, t) l^{(3)}(t) d t,
\end{aligned}
$$


where

$$
K(y, t)= \begin{cases}1, & 2 \gamma+y-\eta \leqslant t \leqslant \eta+y, \\ 0, & \text { elsewhere. }\end{cases}
$$

$K(y, t)$ is the product of two TP-functions, $J(y, t)$ and $M(y, t)$, where

$$
J(y, t)= \begin{cases}1, & 2 \gamma+y-\eta \leqslant t<\infty, \\ 0, & \text { elsewhere }\end{cases}
$$

and

$$
M(y, t)= \begin{cases}1, & -\infty<t \leqslant \eta+y \\ 0, & \text { elsewhere }\end{cases}
$$

Therefore, for $y_{1}<y_{2}, t_{1}<t_{2}$,

$$
K\left(\begin{array}{c}
y_{1}, y_{2} \\
t_{1}, t_{2}
\end{array}\right) \geqslant M\left(y_{1}, t_{2}\right) M\left(y_{2}, t_{1}\right) J\left(\begin{array}{c}
y_{1}, y_{2} \\
t_{1}, t_{2}
\end{array}\right) \geqslant 0,
$$

and so $K(y, t)$ is at least $\mathrm{TP}_{2}$.

Since $l^{(3)}(t)$ changes sign once, by the variation-diminishing theorem, $l^{(2)}(\eta+y)-l^{(2)}(\eta-y)$ has at most one change of sign in $y>0$. However, $l^{(2)}(\eta+y)-l^{(2)}(\eta-y)$ tends to zero as $y$ tends to 0 and as $y$ tends to infinity, the second following from (5.3) and because $l^{(2)}(x)$ has a finite limit as $x$ tends to infinity. Therefore, a change of sign of $l^{(2)}(\eta+y)-l^{(2)}(\eta-y)$ would require $l^{(3)}(x)$ to have two changes of sign. Hence, the second derivative of $g_{r}(\eta+y)-$ $g_{r}(\eta-y)$ does not change sign, and is negative if $l^{(3)}(x)$ changes from negative to positive values, and positive if the change is reversed. Since $g_{r}(\eta+y)-$ $g_{r}(\eta-y)$ is zero when $y$ is zero, this function may change sign no more than once.

Similarly, the proof for $\eta<\gamma$ follows, with the sign of the second derivative of $g_{r}(\eta+y)-g_{r}(\eta-y)$ reversed.

The Pearson Type VI and Type VII distributions with range $(0, \infty)$ satisfy the conditions of Lemma 3.

For the Type VI distribution with density function $f(x)$ of the form

$$
K(x+a)^{-q_{1}} x^{q_{2}}, \quad q_{1}>q_{2}+1>0, a>0,
$$

the moments $\alpha_{r}$ exist for $r<q_{1}-q_{2}-1$. One value of $\gamma$ exists, $\gamma=\log a$, $\left(\log f\left(e^{x}\right)\right)^{(3)}$ changes sign from negative to positive values, and $\left(\log f\left(e^{x}\right)\right)^{(2)}$ has a zero limit at infinity. The value of $r$ corresponding to $\eta_{r}=\log a$ is given by

$$
\frac{1}{2} q_{1}-q_{2}-1
$$

which is thus the unique symmetry value $c$. Thus Theorem III applies, and $L_{r+u, r-u}$ is monotonic in $u$ with a turning point in the type of monotonicity at

$$
r=\frac{1}{2} q_{1}-q_{2}-1 \text {. }
$$


From Lemma $3, g_{r}\left(\eta_{r}+y\right)-g_{r}\left(\eta_{r}-y\right)$ changes from positive to negative values for $\eta_{r}>\gamma$ and hence $\left(\log \alpha_{r}\right)^{(3)}<0$ for $r>c$, while similarly, $\left(\log \alpha_{r}\right)^{(3)}>$ 0 for $r<c$. Therefore, inequality $\mathrm{I}_{1}$ holds for

$$
r<\frac{1}{2} q_{1}-q_{2}-1 \text {, }
$$

and inequality $I_{2}$ for

$$
r>\frac{1}{2} q_{1}-q_{2}-1
$$

For the Type VII distribution with density function $f(x)$ of the form $K\left(c_{0}+c_{2} x^{2}\right)^{-1 / 2 c_{2}}$, truncated to $x>0$, with $c_{0}, c_{2}>0$, the moments $\alpha_{r}$ exist for

$$
r<\frac{1}{c_{2}}-1 \text {. }
$$

The unique value of $\gamma$ is $\frac{1}{2} \log c_{0} / c_{2},\left(\log f\left(e^{x}\right)\right)^{(3)}$ changes sign from negative to positive values, and $\left(\log f\left(e^{x}\right)\right)^{(2)}$ has a zero limit at infinity. The symmetry value

$$
c=\frac{1}{2 c_{2}}-1 \text {, }
$$

and as above, inequality $I_{1}$ holds for

$$
r<\frac{1}{2 c_{2}}-1
$$

and inequality $\mathbf{I}_{2}$ for

$$
r>\frac{1}{2 c_{2}}-1
$$

For the inverse Gaussian, with density of the form $K x^{-3 / 2}$ exp $\lambda(x-\mu)^{2}\left(2 \mu^{2} x\right)^{-1}$, there is one value of $\gamma, \log \mu$, which is a symmetry point, with symmetry value $c=\frac{1}{2}$ but $\left(\log f\left(e^{y}\right)\right)^{(2)} \nrightarrow$ finite limit as $y \rightarrow \infty$. However by direct inspection, $S^{-}\left(g_{r}(\eta+y)-g_{r}(\eta-y)\right)<1$, Theorem III can be applied, and for $r<\frac{1}{2}, \mathrm{I}_{1}$ holds while for $r>\frac{1}{2}, \mathrm{I}_{2}$ holds.

\section{Discussion}

The conditions given here for which the new inequalities hold are conditions on $f(x)$ which basically ensure that $q_{r}(y)=g_{r}\left(\eta_{r}+y\right)-g_{r}\left(\eta_{r}-y\right)$ changes sign in $y$ at most once. In Section 3, the change is in the same direction for all $r$, and $I_{1}$ or $I_{2}$ hold for all possible moment orders. Sections 4 and 5 investigate the changeover properties of symmetry points. For some distributions which do not satisfy the conditions on $f(x)$, direct inspection of $q_{r}(y)$ may give results; for 
example, for the normal with positive mean and negative values truncated, $q_{r}(y)$ changes in $y$ from positive to negative for any $r$, so that $\left(\log \alpha_{r}\right)^{(3)}<0$ and $\mathbf{I}_{2}$ holds.

In the general context, to ensure a given sign for $\mu_{3}$, it appears difficult to go beyond the condition that $h(\mu+x)-h(\mu-x)$ changes sign once only, although this also fixes the signs of the remaining odd central moments. However, the application of this apparently restrictive condition to investigating the monotonicity of $L_{r+u, r-u}$, has given inequalities for the moments of a reasonably wide class of continuous distributions.

\section{Acknowledgements}

This work forms part of my Ph.D. thesis, supervised by Professor S. Lipton at the University of Queensland. My thanks are due to Dr. E. T. White, who drew attention to the problems in te engineering context.

\section{References}

T. Allen (1975), Particle size measurement 2nd ed. (Chapman and Hall, London).

R. E. Barlow, A. W. Marshall and F. Proschan (1963), 'Properties of probability distributions with monotone hazard rate', Ann. Math. Statist. 34, 375-389.

M. H. Belz (1947), Note on the Liapounoff inequality for absolute moments', Ann. Math. Statist. 18, 604-605.

S. Karlin, F. Proschan and R. E. Barlow (1961), 'Moment inequalities of Pólya frequency functions', Pacific J. Math. 11, 1023-1033.

S. Karlin (1968), Total positivity Vol. 1 (Stanford University Press, Stanford).

E. Lukacs (1960), Characteristic functions (Charles Griffen and Co. Ltd., London).

P. A. P. Moran (1969), An introduction to probability theory (Clarendon Press, Oxford).

G. Marsaglia, A. W. Marshall and F. Proschan (1965), 'Moment crossings as related to density crossings', J. Roy. Statist. Soc. Ser. B 27, 91-93.

A. D. Randolph and M. A. Larson (1971), Theory of particulate processes: analysis and techniques of continuous crystallization (Academic Press, New York).

E. T. White (1971), Industrial crystallization (Department of Chemical Engineering, University of Queensland, Brisbane).

Department of Mathematics

University of Queensland

St. Lucia, Queensland 4067

Australia 